\title{
High-Accuracy Joint Position and Orientation Estimation in Sparse 5G mmWave channel
}

\author{
Jukka Talvitie*, Mike Koivisto*, Toni Levanen*, Mikko Valkama*, Giuseppe Destino ${ }^{\dagger}$, Henk Wymeersch ${ }^{\ddagger}$ \\ *Department of Electrical Engineering, Tampere University, Finland \\ ${ }^{\dagger}$ Centre for Wireless Communications, University of Oulu, Finland \\ $\ddagger$ Department of Electrical Engineering, Chalmers University of Technology, Sweden
}

\begin{abstract}
With the emergence of new 5G radio networks, high-accuracy positioning solutions are becoming extensively more important for numerous 5G-enabled applications and radio resource management tasks. In this paper, we focus on 5G mm-wave systems, and propose a method for high-accuracy estimation of the User Equipment (UE) position and antenna orientation. Based on the sparsity of the mm-wave channel, we utilize a compressive sensing approach for estimating the departure and arrival angles as well as the time-of-arrival for each observed radio propagation path. After this, in order to obtain statistical descriptions of the unknown parameters, we analytically derive a set of sampling distributions, which enable utilization of an iterative Gibbs sampling method. As shown by the obtained simulation results, the proposed method is able to achieve centimeter-level positioning accuracy with degree-level orientation accuracy, even in the absence of a line-of-sight path.

Index Terms-Positioning, Orientation estimation, 5G systems, mm-wave, MIMO, Compressed sensing, Gibbs sampling
\end{abstract}

\section{INTRODUCTION}

Utilization of millimeter-wave (mm-wave) communications, at around $30 \mathrm{GHz}$ and beyond carrier frequencies, is expected to introduce high data rate radio links in future 5G systems [1]. Besides large bandwidths, the mm-wave frequencies enable the use of large antenna arrays with dynamic beamforming at both transmitter and receiver side. The mm-wave channel introduces specific sparse characteristics, which can be exploited for channel parameter estimation via compressed sensing as presented in [2]-[6]. Moreover, the particular mm-wave channel properties introduce great potential for high-accuracy User Equipment (UE) positioning, as studied in [6]-[11], where in [9]-[11] also the UE orientation has been considered.

In this paper, we consider UE position and orientation estimation based on a single Base Station (BS) signal, which is one of the key features to overcome limiting factors of the previous generation of mobile network positioning, such as synchronization, scheduling and interference management. By using a distributed-compressed-sensing-based approach, we estimate the Angle-Of-Departure (AOD), Angle-Of-Arrival (AOA) and Time-Of-Arrival (TOA) for each radio propagation

This work was partially supported by the Academy of Finland project FALCON (Grant No. 298781), EU H2020 projects HIGHTS (Grant No. MG3.5a-2014-636537) and 5GCAR, the VINNOVA COPPLAR project, funded under Strategic Vehicle Research and Innovation (Grant No. 2015-04849), as well as the Doctoral Program of the President of Tampere University of Technology, and Business Finland under the projects TAKE-5 and WIVE. path. The estimated AOD, AOA, and TOA are then used for estimating the UE position, the UE orientation, and additionally the scatterer positions for Non-Line-Of-Sight (NLOS) paths.

The proposed novel estimation approach is based on an iterative Gibbs sampling method providing both the parameter estimates and their non-linear likelihood distributions, which have not yet been revealed in the existing literature before. From analytical and system implementation perspective, the proposed approach includes several attractive features. Firstly, it analytically expresses the parameter distributions required in the Gibbs sampling process in order to obtain joint non-linear likelihood distribution of the unknown parameters. Secondly, the proposed approach does not require a priori information on the number of propagation paths or on the availability of the LOS path, which supports the feasibility of the method in a vast set of multipath scenarios. Moreover, the proposed approach is based on a single BS signal, which alleviates requirements for accurate network synchronization. Lastly, the proposed approach can be straightforwardly extended to an uplink scenario, enabling a new set of applicable use cases, such as location-aware radio resource management. As such, the proposed approach can be utilized in a Simultaneous Localization and Mapping (SLAM) setting, or in various tracking scenarios from pedestrians to vehicular navigation.

\section{SYSTEM MODEL}

As a baseline system we consider a Multiple-Input MultipleOutput (MIMO) channel model with a known BS position $\mathbf{p}_{\text {TX }} \in \mathbb{R}^{2}$ with known antenna orientation, and an unknown UE position $\mathbf{p}_{\mathrm{RX}} \in \mathbb{R}^{2}$ with unknown antenna orientation $\theta$. To enable positioning, the BS transmits a sequence of $M$ Orthogonal Frequency-Division Multiplexing (OFDM) symbols on $N$ active subcarriers $\mathbf{x}[m, n]=\left[x_{0}[m, n], \ldots, x_{N_{\mathrm{b}}-1}[m, n]\right]^{T}$, where $m$ and $n$ are OFDM symbol index and the subcarrier index, and $N_{\mathrm{b}}$ is the number of digital TX paths. It is assumed that the channel remains constant during the transmission of $M$ OFDM symbols, which limits the maximum tolerable system mobility. Based on the channel model described in [12], the MIMO channel matrix $\mathbf{H}[n] \in \mathbb{C}^{N_{\mathrm{R}} \times N_{\mathrm{T}}}$ for the $n^{\text {th }}$ subcarrier, with $N_{\mathrm{T}}$ and $N_{\mathrm{R}}$ number of transmit and receive antennas, is written as

$$
\begin{aligned}
\mathbf{H}[n] & =\mathbf{A}_{\mathrm{R}}[n] \boldsymbol{\Gamma}[n] \mathbf{A}_{\mathrm{T}}^{H}[n], \text { where } \\
\mathbf{A}_{\mathrm{T}}[n] & =\left[\mathbf{a}_{\mathrm{T}, n}\left(\varphi_{\mathrm{T}, 0}\right), \ldots, \mathbf{a}_{\mathrm{T}, n}\left(\varphi_{\mathrm{T}, K-1}\right)\right],
\end{aligned}
$$




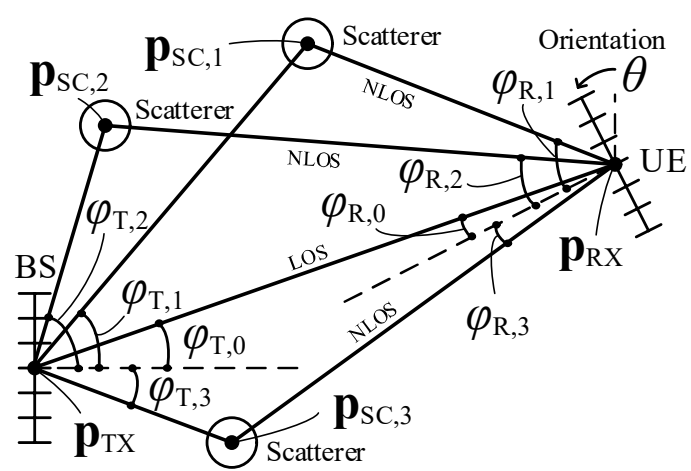

Fig. 1. An example scenario of the LOS path and 3 NLOS paths including the AOD $\varphi_{\mathrm{T}, k}$ and AOA $\varphi_{\mathrm{R}, k}$ for each path $k$, where the AOAs are affected by the unknown orientation of the UE.

and similarly for $\mathbf{A}_{\mathrm{R}}[n]$, are steering vector matrices introducing the $\operatorname{AOD} \varphi_{\mathrm{R}, k}$ and $\mathrm{AOA} \varphi_{\mathrm{T}, k}$ for each path $k=0, \ldots, K-1$, where $K$ is the number of observable paths. Moreover, $\boldsymbol{\Gamma}[n]=\operatorname{diag}\left(\gamma_{0}, \ldots, \gamma_{K-1}\right)$ is a diagonal channel coefficient matrix whose $k^{\text {th }}$ diagonal element is given as

$$
\gamma_{k}=\sqrt{N_{\mathrm{T}} N_{\mathrm{R}}} \frac{h_{k}}{\sqrt{\xi_{k}}} e^{-j 2 \pi n \tau_{k} /\left(N T_{s}\right)},
$$

where $h_{k}, \xi_{k}$ and $\tau_{k}$ are the complex channel coefficient, the path loss coefficient and the TOA of the $k^{\text {th }}$ path, respectively. Here $T_{s}=1 / B$ denotes the sample duration and $B$ is the bandwidth of the transmitted signal. In addition, throughout the paper we consider antenna structures based on a Uniform Linear Array (ULA) with antenna separation of $d_{\mathrm{ant}}$. Therefore, the steering vector $\mathbf{a}_{\mathrm{T}, n}\left(\varphi_{\mathrm{T}, k}\right)$ (and similarly for $\mathbf{a}_{\mathrm{R}, n}\left(\varphi_{\mathrm{R}, k}\right)$ ), given in (1), can be described as

$$
\mathbf{a}_{\mathrm{T}, n}\left(\varphi_{\mathrm{T}, k}\right)=\frac{1}{\sqrt{N_{\mathrm{T}}}}\left[e^{-j \frac{N_{\mathrm{T}}-1}{2} \Phi\left(\varphi_{\mathrm{T}, k}\right)}, \ldots, e^{j \frac{N_{\mathrm{T}}-1}{2} \Phi\left(\varphi_{\mathrm{T}, k}\right)}\right]^{T}
$$

where $\Phi\left(\varphi_{\mathrm{T}, k}\right)=2 \pi d_{\text {ant }} / \lambda_{n} \sin \left(\varphi_{\mathrm{T}, k}\right)$ is the electrical AOD for the $k^{\text {th }}$ path, and $\lambda_{n}$ is the wavelength of the $n^{\text {th }}$ subcarrier. In Fig. 1, an example scenario with the LOS path and 3 NLOS paths is illustrated.

Assuming that the transmitted OFDM symbols are embedded with an appropriate length cyclic prefix $(\mathrm{CP})$, there is no intersymbol-interference in the received signal. Hence, with ideal sampling timing and $\mathrm{CP}$ removal, the received spatial sample vector of the $m^{\text {th }}$ OFDM symbol and $n^{\text {th }}$ subcarrier, after taking the Fast Fourier Transform (FFT), is given as

$$
\mathbf{y}[m, n]=\mathbf{H}[n] \mathbf{F}[m, n] \mathbf{x}[m, n]+\mathbf{n}[m, n],
$$

where $\mathbf{n}[m, n]$ is complex Gaussian noise and $\mathbf{F}[m, n] \in$ $\mathbb{C}^{N_{\mathrm{T}} \times N_{\mathrm{b}}}$ is a beamforming matrix, known by the UE.

\section{ChANNEL PARAMETER ESTIMATION}

In order to harness the mm-wave channel sparsity for channel estimation, we determine a transformation from the MIMO channel matrix to an angular domain beamspace matrix as

$$
\mathbf{H}_{\varphi}[n]=\mathbf{U}_{\mathrm{R}}^{H} \mathbf{H}[n] \mathbf{U}_{\mathrm{T}} \in \mathbb{C}^{N_{\mathrm{R}} \times N_{\mathrm{T}}},
$$

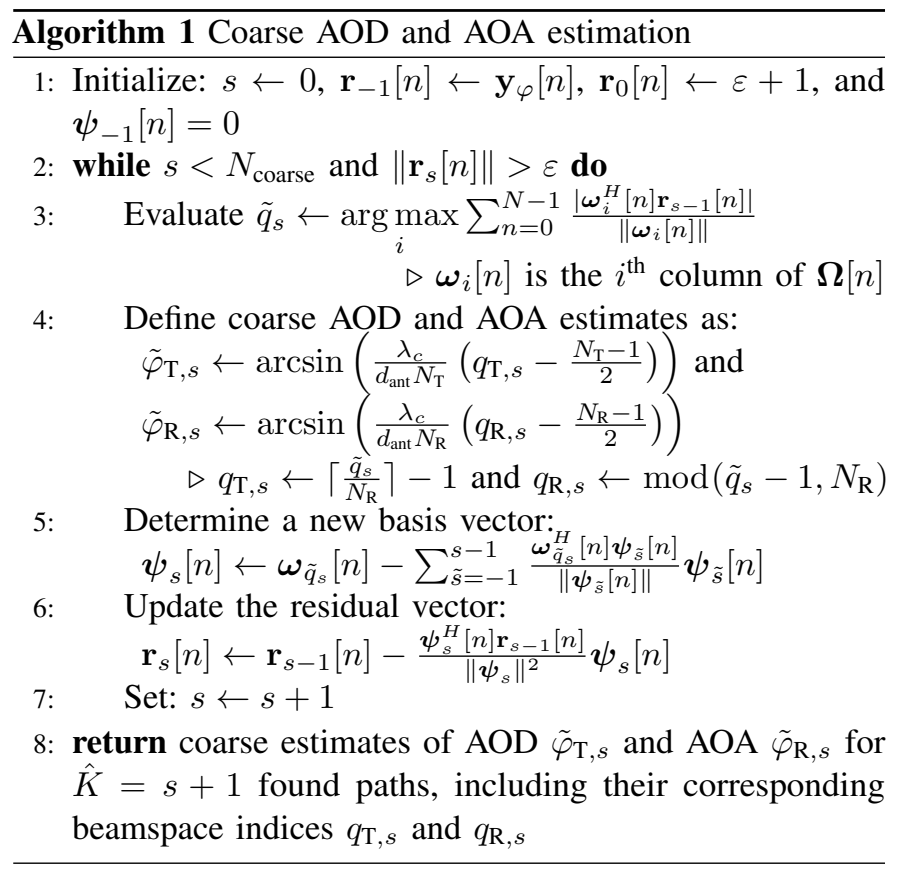

where $\mathbf{U}_{\mathrm{T}}$ and $\mathbf{U}_{\mathrm{R}}$ are chosen transformation matrices. For example, with the considered ULA model, by spanning the beamspace with uniformly sampled angles, $\mathbf{U}_{\mathrm{T}}$ (and similarly $\mathrm{U}_{\mathrm{R}}$ ) can be defined as

$$
\begin{aligned}
\mathbf{U}_{\mathrm{T}} & =\left[\mathbf{u}_{\mathrm{T}}\left(\nu_{0}\right), \ldots, \mathbf{u}_{\mathrm{T}}\left(\nu_{N_{\mathrm{T}}-1}\right)\right], \text { where } \\
\mathbf{u}_{\mathrm{T}}\left(\nu_{l}\right) & =\left[e^{-j 2 \pi \frac{N_{\mathrm{T}}-1}{2} \nu_{l}}, \ldots, e^{j 2 \pi \frac{N_{\mathrm{T}}-1}{2} \nu_{l}}\right]^{T}, \text { and } \\
\nu_{l} & =-\left(N_{\mathrm{T}}-1\right) /\left(2 N_{\mathrm{T}}\right)+l / N_{\mathrm{T}} .
\end{aligned}
$$

Consequently, the beamspace angle resolution for the AOD and AOA are defined as $\kappa_{\mathrm{T}}=1 / N_{\mathrm{T}}$ and $\kappa_{\mathrm{R}}=1 / N_{\mathrm{R}}$. Now, based on (4) and (5), the vectorization of the received samples over the $M$ OFDM symbols results in

$$
\begin{aligned}
\mathbf{y}_{\varphi}[n] & =\boldsymbol{\Omega}[n] \mathbf{h}_{\varphi}[n]+\mathbf{n}_{\varphi}[n], \text { where } \\
\boldsymbol{\Omega}[n] & =\left[\boldsymbol{\Omega}_{0, n}, \ldots, \boldsymbol{\Omega}_{M-1, n}\right]^{T} \text { with } \\
\boldsymbol{\Omega}_{m, n} & =\left(\mathbf{U}_{\mathrm{T}}^{H} \mathbf{F}[m, n] \mathbf{x}[m, n]\right)^{T} \otimes \mathbf{U}_{\mathrm{R}}, \text { and } \\
\mathbf{h}_{\varphi}[n] & =\operatorname{vec}\left(\mathbf{H}_{\varphi}[n]\right),
\end{aligned}
$$

where $\mathbf{h}_{\varphi}[n]$ is now a nearly sparse channel vector, and $\boldsymbol{\Omega}[n]$ is a dictionary (or sensing) matrix whose columns depend on different combinations of channel AOD and AOA values.

Since $\mathbf{h}_{\varphi}[n]$ in (7) has a common support over separate subcarriers, the channel parameter estimation is performed based on Distributed Compressed Sensing - Simultaneous Orthogonal Matching Pursuit (DCS-SOMP) method presented in [13]. Considering the beamspace representation shown in (7), the used estimation approach is described in Algorithm 1, where the design parameters $N_{\text {coarse }}$ and $\varepsilon$ define the maximum number of detected paths and the path detection sensitivity, respectively. In order to increase the estimation accuracy, we use an iterative refinement method presented in [10], where the beamspace resolution is iteratively increased with inbuilt 
convergence stability. By first defining a desired number of resolution increment steps $N_{\text {fine }}$, the refinement method can be carried out for each found path, as described in Algorithm 2.

As shown in [9] and [13], the $k^{\text {th }}$ path TOA estimate can be obtained by using QR-factorization of the estimated sparse dictionary matrix, whose columns are obtained from the Algorithm 2 as $\hat{\boldsymbol{\Omega}}[n]=\left[\hat{\boldsymbol{\omega}}_{0}[n], \ldots, \hat{\boldsymbol{\omega}}_{\hat{K}-1}[n]\right]$. Moreover, by evaluating the QR-factorization $\hat{\boldsymbol{\Omega}}[n]=\mathbf{Q}[n] \mathbf{R}[n]$, the estimated channel vector can be written as

$$
\begin{aligned}
\hat{\mathbf{h}}[n] & =\mathbf{R}^{-1}[n]\left[\tilde{\beta}_{0}[n], \ldots, \tilde{\beta}_{\hat{K}-1}[n]\right]^{T}, \text { where } \\
\tilde{\beta}_{k}[n] & =\frac{\hat{\boldsymbol{\omega}}_{k}^{H}[n] \mathbf{y}_{\varphi}[n]}{\left\|\hat{\boldsymbol{\omega}}_{k}[n]\right\|^{2}} .
\end{aligned}
$$

Based on the used channel model, described in (1), the Least Squares (LS) estimate of the $k^{\text {th }}$ path TOA can be obtained as

$$
\begin{gathered}
\hat{\tau}_{k}=\underset{\tau_{k}}{\arg \max }\left|\zeta^{H}\left(\tau_{k}\right)\left[\hat{h}_{k}[0], \ldots, \hat{h}_{k}[N-1]\right]^{T}\right|^{2} \text { with } \\
\zeta\left(\tau_{k}\right)=\left[1, \ldots, e^{-j 2 \pi(N-1) \tau_{k} /\left(N T_{s}\right)}\right]^{T},
\end{gathered}
$$

where $\hat{h}_{k}[n]$ is the $k^{\text {th }}$ element of $\hat{\mathbf{h}}[n]$ given in (8). Now, the TOA estimate $\hat{\tau}_{k}$ can be found by searching over different values of $\tau_{k}$ and choosing the one maximizing the function.

\section{PROPOSED POSITION AND ORIENTATION ESTIMATION}

The UE position $\mathbf{p}_{\mathrm{RX}}$ and UE orientation $\theta$ are estimated by using iterative sampling techniques based on the estimates of AOD, AOA and TOA, obtained in Section III. If the LOS path is detected, it is denoted with the path index $k=0$. Moreover, we convert the $k^{\text {th }}$ path TOA-estimate to a distance estimate as $\hat{d}_{k}=c \hat{\tau}_{k}$, where $c$ is the speed of light. In the considered approach, we also utilize the parameter estimate covariances, which are not provided by the DCS-SOMP algorithm. Thus, the covariance matrix $\boldsymbol{\Sigma}_{k} \in \mathbb{R}^{3 \times 3}$ for the $k^{\text {th }}$ path AOD, AOA and TOA-based distance estimates is given as

$$
\boldsymbol{\Sigma}_{k}=J^{-1}(\hat{\boldsymbol{\eta}})+\operatorname{diag}\left\{\tilde{\sigma}_{\mathrm{AOD}}^{2}, \tilde{\sigma}_{\mathrm{AOA}}^{2}, \tilde{\sigma}_{\mathrm{d}}^{2}\right\},
$$

where $\hat{\boldsymbol{\eta}}_{k}=\left[\hat{\varphi}_{\mathrm{T}, k}, \hat{\varphi}_{\mathrm{R}, k}, \hat{d}_{k}\right]^{T}$ is the vector of the estimated channel parameters, and $J\left(\hat{\boldsymbol{\eta}}_{k}\right)$ is the Fisher information matrix, whose elements are derived based on [9, Appendix A]. In addition, the variance components $\tilde{\sigma}_{\text {AOD }}^{2}, \tilde{\sigma}_{\text {AOA }}^{2}$ and $\tilde{\sigma}_{\mathrm{d}}^{2}$ incorporate the effect of an estimate offset due to the discrete estimation space for the AOD, AOA, and TOA, respectively.

The proposed UE position and UE orientation estimation is based on an iterative Gibbs sampling process, where each estimated parameter is iteratively sampled by conditioning with the currently available samples of the other parameters. Although the convergence of Gibbs sampling can be occasionally slow, as discussed in [14], it enables a straightforward sampling implementation without extensive algorithm parameterization by directly utilizing conditional distributions of the unknown parameters. Next, in this section, we analytically derive sampling distributions and provide the means for sampling of each estimated parameter. Based on these, the overall sampling process for estimating the unknown parameters is described

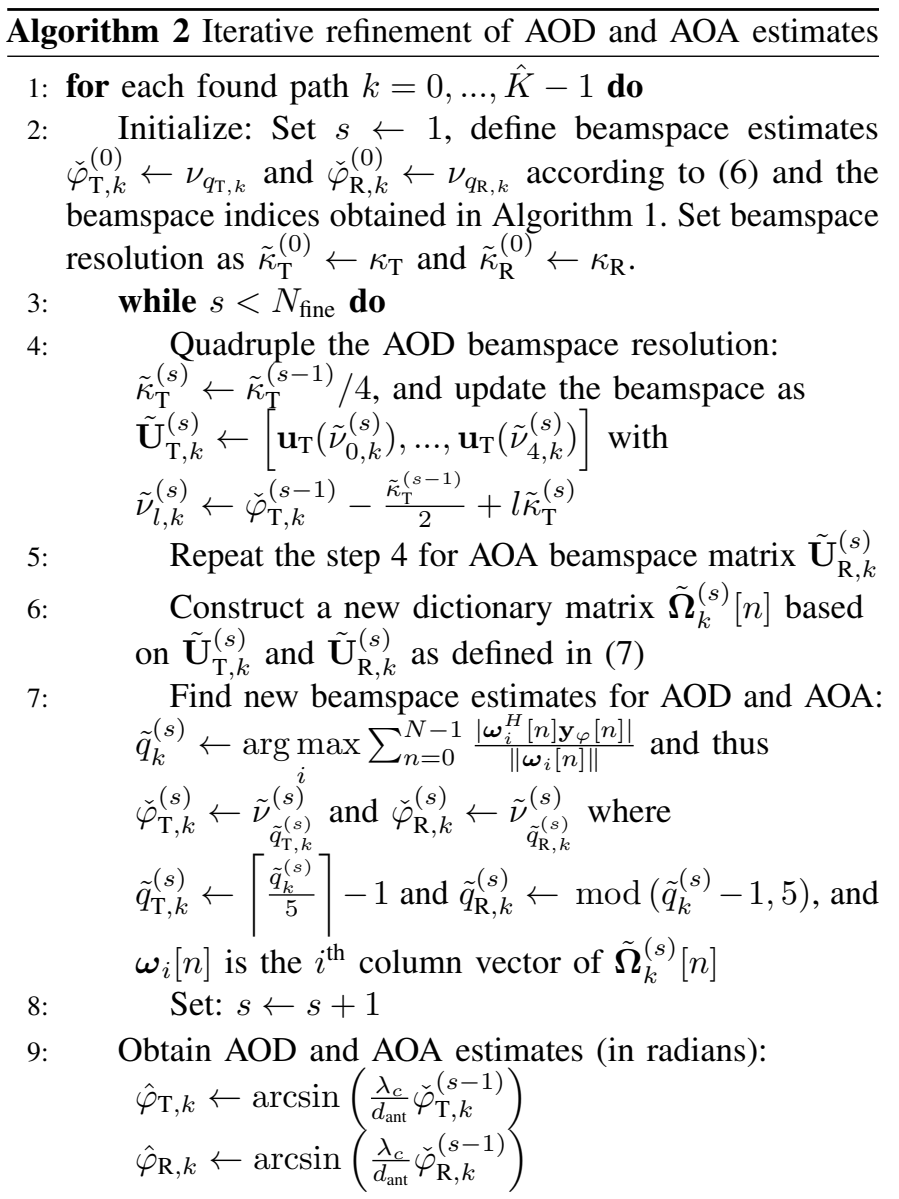

10: return Estimates of AOD $\hat{\varphi}_{\mathrm{T}, k}$ and AOA $\hat{\varphi}_{\mathrm{R}, k}$, and the latest column vector $\hat{\boldsymbol{\omega}}_{k}[n] \leftarrow \boldsymbol{\omega}_{\tilde{q}_{k}^{(s-1)}}[n]$ for each path $k$

in Algorithm 3, where $N_{\text {samp }}$ and $N_{\text {burn-in }}$ are the number of obtained samples and the length of burn-in period reducing the effect of parameter initialization, which can be done based on the geometrical properties of the system, as studied in [10]. An illustration of a sampling process outcome is shown in Fig. 2 , where the system geometry is equivalent with the geometry used in evaluating the positioning performance in Section V.

\section{A. Sampling of Scatterer Positions}

First we obtain the scatterer position sample $\mathbf{p}_{\mathrm{SC}, k}^{(i)}$ for each found NLOS path $k \in \Theta_{\mathrm{NLOS}}$, where $i$ is the sample index and $\Theta_{\text {NLOS }}$ is the set of NLOS path indices. We consider scatterer positions of different paths independent and perform the sampling process for each path separately. Based on the Gibbs sampling principle, the target is to obtain a $k^{\text {th }}$ path scatterer position sample $\mathbf{p}_{\mathrm{SC}, \mathrm{k}}^{(i)}$ by conditioning with the most recent samples of the other unknown parameters: the UE position sample $\mathbf{p}_{\mathrm{RX}}^{(i-1)}$ and the UE orientation sample $\theta^{(i-1)}$. Thus, the $i^{\text {th }}$ sample of the $k^{\text {th }}$ path scatterer position is given as

$$
\mathbf{p}_{\mathrm{SC}, k}^{(i)} \sim p\left(\mathbf{p}_{\mathrm{SC}, k} \mid \mathbf{p}_{\mathrm{RX}}^{(i-1)}, \theta^{(i-1)}, \hat{\boldsymbol{\eta}}_{k}\right) .
$$

Sampling from the above-described conditional probability 


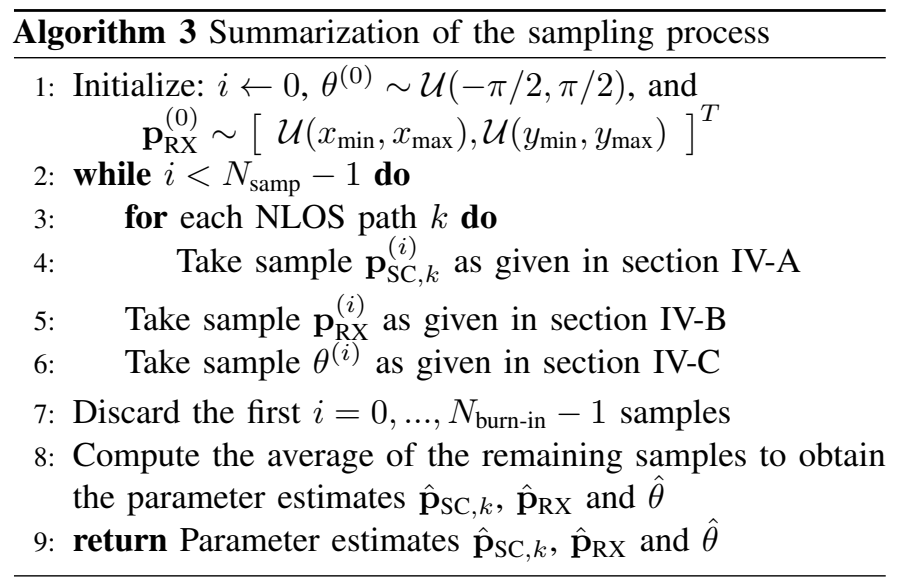

distribution is not directly feasible. However, based on the Bayes' rule, the distribution can be rewritten as

$$
\begin{aligned}
& p\left(\mathbf{p}_{\mathrm{SC}, k} \mid \mathbf{p}_{\mathrm{RX}}^{(i-1)}, \theta^{(i-1)}, \hat{\boldsymbol{\eta}}_{k}\right) \propto \\
& p\left(\hat{\boldsymbol{\eta}}_{k} \mid \mathbf{p}_{\mathrm{SC}, k}, \mathbf{p}_{\mathrm{RX}}^{(i-1)}, \theta^{(i-1)}\right) p\left(\mathbf{p}_{\mathrm{SC}, k} \mid \mathbf{p}_{\mathrm{RX}}^{(i-1)}, \theta^{(i-1)}\right) \\
& =p\left(\hat{\boldsymbol{\eta}}_{k} \mid \mathbf{p}_{\mathrm{SC}, k}, \mathbf{p}_{\mathrm{RX}}^{(i-1)}, \theta^{(i-1)}\right) p\left(\mathbf{p}_{\mathrm{SC}, k}\right) .
\end{aligned}
$$

By assuming no a priori information, the distribution $p\left(\mathbf{p}_{\mathrm{SC}, k}\right)$ can be considered flat. Moreover, assuming that the channel parameter estimates are Gaussian distributed, the normalized log-likelihood for an arbitrary scatterer position $\breve{\mathrm{p}}_{\mathrm{SC}, k}$ can be written as

$$
\begin{gathered}
\log p\left(\hat{\boldsymbol{\eta}}_{k}, \boldsymbol{\Sigma}_{k} \mid \breve{\mathbf{p}}_{\mathrm{SC}, k}, \mathbf{p}_{\mathrm{RX}}^{(i-1)}, \theta^{(i-1)}\right) \propto \\
-\frac{1}{2}\left(\hat{\boldsymbol{\eta}}_{k}-\breve{\boldsymbol{\eta}}_{\mathrm{SC}, k}\right)^{T} \boldsymbol{\Sigma}_{k}^{-1}\left(\hat{\boldsymbol{\eta}}_{k}-\breve{\boldsymbol{\eta}}_{\mathrm{SC}, k}\right)
\end{gathered}
$$

where $\breve{\eta}_{\mathrm{SC}, k}=\left[\breve{\varphi}_{\mathrm{SC}, \mathrm{T}, k}, \breve{\varphi}_{\mathrm{SC}, \mathrm{R}, k}, \breve{d}_{\mathrm{SC}, k}\right]$ is a vector of the AOD, AOA and propagation distance, given the scatterer position $\breve{\mathbf{p}}_{\mathrm{SC}, k}$, whose elements are defined as

$$
\begin{aligned}
\breve{\varphi}_{\mathrm{SC}, \mathrm{T}, k} & =\arctan \left(\frac{\breve{\mathbf{p}}_{(\mathrm{SC}, k), \mathrm{y}}-\mathbf{p}_{\mathrm{TX}, \mathrm{y}}}{\breve{\mathbf{p}}_{(\mathrm{SC}, k), \mathrm{x}}-\mathbf{p}_{\mathrm{TX}, \mathrm{x}}}\right) \\
\breve{\varphi}_{\mathrm{SC}, \mathrm{R}, k} & =-\arctan \left(\frac{\mathbf{p}_{\mathrm{RX}, \mathrm{y}}^{(i-1)}-\breve{\mathbf{p}}_{(\mathrm{SC}, k), \mathrm{y}}}{\mathbf{p}_{\mathrm{RX}, \mathrm{x}}^{(i-1)}-\breve{\mathbf{p}}_{(\mathrm{SC}, k), \mathrm{x}}}\right)+\theta^{(i-1)} \\
\breve{d}_{\mathrm{SC}, k} & =\left\|\mathbf{p}_{\mathrm{TX}}-\breve{\mathbf{p}}_{\mathrm{SC}, k}\right\|+\left\|\mathbf{p}_{\mathrm{RX}}^{(i-1)}-\breve{\mathbf{p}}_{\mathrm{SC}, k}\right\|
\end{aligned}
$$

where $\breve{\mathbf{p}}_{(\mathrm{SC}, k), \mathrm{x}}, \breve{\mathbf{p}}_{(\mathrm{SC}, k), \mathrm{y}}, \mathbf{p}_{\mathrm{TX}, \mathrm{x}}, \mathbf{p}_{\mathrm{TX}, \mathrm{y}}, \mathbf{p}_{\mathrm{RX}, \mathrm{x}}^{(i-1)}$ and $\mathbf{p}_{\mathrm{RX}, \mathrm{y}}^{(i-1)}$, denote the $\mathrm{x}$ coordinates and $\mathrm{y}$ coordinates for the given scatterer position, known BS position, and sampled UE position, respectively.

In order to obtain a sample of the scatterer position $\mathbf{p}_{(\mathrm{SC}, k)}^{(i)}$ from (13), we utilize the rejection sampling principle [14]. For this, we notice that the considered log-likelihood function in (13) is normalized so that the target set of the linear scale likelihood function is defined as $[0,1]$. Now, according to the rejection sampling principle, we draw a candidate sample of $\mathbf{p}_{\mathrm{SC}, k}^{(i)}$ from a uniform distribution as

$$
\mathbf{p}_{\mathrm{SC}, k}^{(i)} \sim\left[\mathcal{U}\left(x_{\min }, x_{\max }\right) \mathcal{U}\left(y_{\min }, y_{\max }\right)\right]^{T}
$$

where the limits $x_{\min }, x_{\max }, y_{\min }$, and $y_{\max }$ are defined so that the sampling range covers the area of the likelihood distribution, where it has substantial significance. Next, we draw a likelihood sample $\rho$ from the target set of the likelihood function as $\rho \sim \mathcal{U}(0,1)$ and compare that to the likelihood as

$$
\rho<p\left(\hat{\boldsymbol{\eta}}_{k} \mid \mathbf{p}_{\mathrm{SC}, k}^{(i)}, \mathbf{p}_{\mathrm{RX}}^{(i-1)}, \theta^{(i-1)}\right),
$$

where the likelihood function is evaluated by substituting $\breve{\mathbf{p}}_{\mathrm{SC}, k} \leftarrow \mathbf{p}_{\mathrm{SC}, k}^{(i)}$ in (13). If the inequality holds, the current sample is consider valid, and we move on sampling of the next parameter in the Gibbs sampling process. Otherwise, we re-sample $\rho$ and $\mathbf{p}_{\mathrm{SC}, k}$ until the condition in (16) is fulfilled.

\section{B. Sampling of UE Position}

By following the Gibbs sampling principle, the UE position sample is acquired from the conditional distribution given as

$$
\mathbf{p}_{\mathrm{RX}}^{(i)} \sim p\left(\mathbf{p}_{\mathrm{RX}} \mid \mathbf{p}_{\mathrm{SC}, k}^{(i)}, \theta^{(i-1)},\left\{\hat{\boldsymbol{\eta}}_{k} \mid k \in \Theta_{\text {allpaths }}\right\}\right),
$$

where $\Theta_{\text {allpaths }}=\{0, \ldots, K-1\}$ defines all path indices, including both the possible LOS path and NLOS paths. Based on the Bayes' rule, and assuming no a priori information on the UE position, the UE position sample can be obtained from the likelihood distribution $p\left(\left\{\hat{\boldsymbol{\eta}}_{k} \mid k \in \Theta_{\text {allpaths }}\right\} \mid \mathbf{p}_{\mathrm{SC}, k}^{(i)}, \theta^{(i-1)}, \mathbf{p}_{\mathrm{RX}}\right)$. Again, by considering Gaussian distributed channel parameter estimates, the normalized log-likelihood function for an arbitrary selected UE position $\breve{\mathbf{p}}_{\mathrm{RX}}$ can be written as

$$
\begin{aligned}
& \quad \log p\left(\left\{\hat{\boldsymbol{\eta}}_{k} \mid k \in \Theta_{\text {allpaths }}\right\} \mid \breve{\mathbf{p}}_{\mathrm{RX}}, \mathbf{p}_{\mathrm{SC}, k}^{(i)}, \theta^{(i-1)}\right)= \\
& \quad \log p_{\mathrm{LOS}}\left(\hat{\boldsymbol{\eta}}_{0} \mid \breve{\mathbf{p}}_{\mathrm{RX}}, \theta^{(i-1)}\right) \\
& +\log p_{\mathrm{NLOS}}\left(\left\{\hat{\boldsymbol{\eta}}_{k} \mid k \in \Theta_{\mathrm{NLOS}}\right\} \mid \breve{\mathbf{p}}_{\mathrm{RX}}, \mathbf{p}_{\mathrm{SC}, k}^{(i)}, \theta^{(i-1)}\right)
\end{aligned}
$$

where $\Theta_{\text {NLOS }}$ is the set of NLOS path indices. Moreover, the likelihood related to a possibly detected LOS path is given as

$$
\begin{gathered}
\log p_{\mathrm{LOS}}\left(\hat{\boldsymbol{\eta}}_{0} \mid \breve{\mathbf{p}}_{\mathrm{RX}}, \theta^{(i-1)}\right) \propto \\
-\frac{1}{2}\left(\hat{\boldsymbol{\eta}}_{0}-\breve{\boldsymbol{\eta}}_{\mathrm{RX}, 0}\right)^{T} \boldsymbol{\Sigma}_{0}^{-1}\left(\hat{\boldsymbol{\eta}}_{0}-\breve{\boldsymbol{\eta}}_{\mathrm{RX}, 0}\right) \\
\text { where } \breve{\boldsymbol{\eta}}_{\mathrm{RX}, 0}=\left[\breve{\varphi}_{\mathrm{RX}, \mathrm{T}, 0}, \breve{\varphi}_{\mathrm{RX}, \mathrm{R}, 0}, \breve{d}_{\mathrm{RX}, 0}\right]^{T} \text { with } \\
\breve{\varphi}_{\mathrm{RX}, \mathrm{T}, 0}=\arctan \left(\frac{\breve{\mathbf{p}}_{\mathrm{RX}, \mathrm{y}}-\mathbf{p}_{\mathrm{TX}, \mathrm{y}}}{\breve{\mathbf{p}}_{\mathrm{RX}, \mathrm{x}}-\mathbf{p}_{\mathrm{TX}, \mathrm{x}}}\right) \\
\breve{\varphi}_{\mathrm{RX}, \mathrm{R}, 0}=-\breve{\varphi}_{\mathrm{RX}, \mathrm{T}, 0}+\theta^{(i-1)} \\
\breve{d}_{\mathrm{RX}, 0}=\left\|\mathbf{p}_{\mathrm{TX}}-\breve{\mathbf{p}}_{\mathrm{RX}}\right\|,
\end{gathered}
$$

and the likelihood describing the NLOS paths is given as

$$
\begin{aligned}
& \log p_{\mathrm{NLOS}}\left(\left\{\hat{\boldsymbol{\eta}}_{k} \mid k \in \Theta_{\mathrm{NLOS}}\right\} \mid \breve{\mathbf{p}}_{\mathrm{RX}}, \mathbf{p}_{\mathrm{SC}, k}^{(i)}, \theta^{(i-1)}\right) \propto \\
& -\frac{1}{2} \sum_{k \in \Theta_{\mathrm{NLOS}}}\left(\hat{\boldsymbol{\eta}}_{k}^{(2: 3)}-\breve{\boldsymbol{\eta}}_{\mathrm{RX}, k}\right)^{T} \boldsymbol{\Sigma}_{k}^{(2: 3)^{-1}}\left(\hat{\boldsymbol{\eta}}_{k}^{(2: 3)}-\breve{\boldsymbol{\eta}}_{\mathrm{RX}, k}\right)
\end{aligned}
$$

where $\hat{\boldsymbol{\eta}}_{k}^{(2: 3)}$ denotes taking only the $2^{\text {nd }}$ and $3^{\text {rd }}$ vector element from $\hat{\boldsymbol{\eta}}_{k}$, and $\boldsymbol{\Sigma}_{k}^{(2: 3)}$ denotes a corresponding submatrix of $\boldsymbol{\Sigma}_{k}$ with only the $2^{\text {nd }}$ and $3^{\text {rd }}$ row and column. In addition, the vector with the AOA and propagation distance, given the UE 
position $\breve{\mathbf{p}}_{\mathrm{RX}}$, is defined as $\breve{\boldsymbol{\eta}}_{\mathrm{RX}, k}=\left[\breve{\varphi}_{\mathrm{RX}, \mathrm{R}, k}, \breve{d}_{\mathrm{RX}, k}\right]^{T}$ with

$$
\begin{gathered}
\breve{\varphi}_{\mathrm{RX}, \mathrm{R}, k}=-\arctan \left(\frac{\breve{\mathbf{p}}_{\mathrm{RX}, \mathrm{y}}-\mathbf{p}_{(\mathrm{SC}, k), \mathrm{y}}^{(i)}}{\breve{\mathbf{p}}_{\mathrm{RX}, \mathrm{x}}-\mathbf{p}_{(\mathrm{SC}, k), \mathrm{x}}^{(i)}}\right)+\theta^{(i-1)} \\
\breve{d}_{\mathrm{RX}, k}=\left\|\mathbf{p}_{\mathrm{TX}}-\mathbf{p}_{\mathrm{SC}, k}^{(i)}\right\|+\left\|\breve{\mathbf{p}}_{\mathrm{RX}}-\mathbf{p}_{\mathrm{SC}, k}^{(i)}\right\| .
\end{gathered}
$$

For obtaining a UE position sample from the above-described likelihood, we use the same rejection sampling principle as with the scatterer position sampling, and draw the UE position sample $\mathbf{p}_{\mathrm{RX}}^{(i)}$ and a separate likelihood sample $\rho$ as

$$
\mathbf{p}_{\mathrm{RX}}^{(i)} \sim\left[\begin{array}{l}
\mathcal{U}\left(x_{\min }, x_{\max }\right) \\
\mathcal{U}\left(y_{\min }, y_{\max }\right)
\end{array}\right] \text { and } \rho \sim \mathcal{U}(0,1) .
$$

Now, based on the rejection sampling principle, we accept the drawn UE position sample, if

$$
\rho<p\left(\left\{\hat{\boldsymbol{\eta}}_{k} \mid k \in \Theta_{\text {allpaths }}\right\} \mid \mathbf{p}_{\mathrm{SC}, k}^{(i)}, \mathbf{p}_{\mathrm{RX}}^{(i)}, \theta^{(i-1)}\right),
$$

where the likelihood is calculated by substituting $\breve{\mathbf{p}}_{\mathrm{RX}} \leftarrow \mathbf{p}_{\mathrm{RX}}^{(i)}$ in (18). Otherwise, we draw new samples until (24) is fulfilled.

\section{Sampling of UE Orientation}

The last sampled unknown parameter is the UE orientation. Similar to the sampling of scatterer and UE positions, the UE orientation sample is taken from the distribution conditioned by the most recent samples of other parameters as

$$
\theta^{(i)} \sim p\left(\theta \mid \mathbf{p}_{\mathrm{SC}, k}^{(i)}, \mathbf{p}_{\mathrm{RX}}^{(i)},\left\{\hat{\boldsymbol{\eta}}_{k} \mid k \in \Theta_{\text {allpaths }}\right\}\right) .
$$

By following the Bayes' rule without any a priori information, the UE orientation sample can be obtained from a likelihood distribution, defined for an arbitrary UE orientation $\breve{\theta}$ as

$$
\begin{aligned}
& \log p\left(\left\{\hat{\boldsymbol{\eta}}_{k} \mid k \in \Theta_{\text {allpaths }}\right\} \mid \breve{\theta}, \mathbf{p}_{\mathrm{SC}, k}^{(i)}, \mathbf{p}_{\mathrm{RX}}^{(i)}\right)= \\
& \quad \log p_{\mathrm{LOS}}\left(\hat{\boldsymbol{\eta}}_{0} \mid \mathbf{p}_{\mathrm{RX}}^{(i)}, \breve{\theta}\right) \\
& +\log p_{\mathrm{NLOS}}\left(\left\{\hat{\boldsymbol{\eta}}_{k} \mid k \in \Theta_{\mathrm{NLOS}}\right\} \mid \mathbf{p}_{\mathrm{SC}, k}^{(i)}, \mathbf{p}_{\mathrm{RX}}^{(i)}, \breve{\theta}\right) .
\end{aligned}
$$

Now, the likelihood for a detected LOS path is given as

$$
\log p_{\mathrm{LOS}}\left(\hat{\boldsymbol{\eta}}_{0} \mid \mathbf{p}_{\mathrm{RX}}^{(i)}, \breve{\theta}\right) \propto \frac{\left(\hat{\boldsymbol{\eta}}_{0}^{(2)}-\breve{\varphi}_{\theta, \mathrm{R}, 0}\right)^{2}}{2 \boldsymbol{\Sigma}_{0}^{(2)}}
$$

where $\hat{\boldsymbol{\eta}}_{k}^{(2)}$ and $\boldsymbol{\Sigma}_{k}^{(2)}$ denote the $2^{\text {nd }}$ vector element and $2^{\text {nd }}$ diagonal element of $\hat{\boldsymbol{\eta}}_{k}$ and $\boldsymbol{\Sigma}_{k}$, respectively, and

$$
\breve{\varphi}_{\theta, \mathrm{R}, 0}=-\arctan \left(\frac{\mathbf{p}_{\mathrm{RX}, \mathrm{y}}^{(i)}-\mathbf{p}_{\mathrm{TX}, \mathrm{y}}}{\mathbf{p}_{\mathrm{RX}, \mathrm{x}}^{(i)}-\mathbf{p}_{\mathrm{TX}, \mathrm{x}}}\right)+\breve{\theta} .
$$

Furthermore, the corresponding likelihood for detected NLOS paths is defined as

$$
\begin{gathered}
\log p_{\mathrm{NLOS}}\left(\left\{\hat{\boldsymbol{\eta}}_{k} \mid k \in \Theta_{\mathrm{NLOS}}\right\} \mid \mathbf{p}_{\mathrm{SC}, k}^{(i)}, \mathbf{p}_{\mathrm{RX}}^{(i)}, \breve{\theta}\right) \propto \\
-\sum_{k \in \Theta_{\mathrm{NLOS}}} \frac{\left(\hat{\boldsymbol{\eta}}_{k}^{(2)}-\breve{\varphi}_{\theta, \mathrm{R}, k}\right)^{2}}{2 \boldsymbol{\Sigma}_{k}^{(2)}, \text { where }} \\
\breve{\varphi}_{\theta, \mathrm{R}, k}=-\arctan \left(\frac{\mathbf{p}_{\mathrm{RX}, \mathrm{y}}^{(i)}-\mathbf{p}_{(\mathrm{SC}, k), \mathrm{y}}^{(i)}}{\mathbf{p}_{\mathrm{RX}, \mathrm{x}}^{(i)}-\mathbf{p}_{(\mathrm{SC}, k), \mathrm{x}}^{(i)}}\right)+\breve{\theta}
\end{gathered}
$$

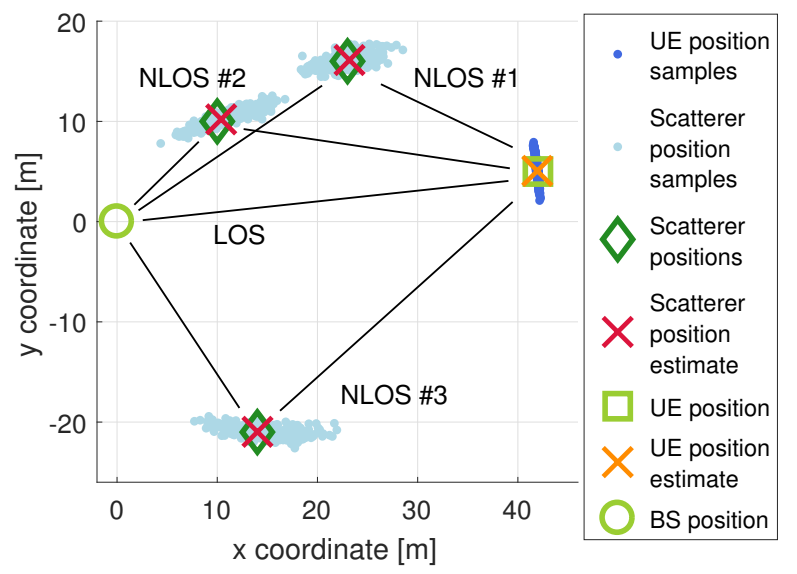

Fig. 2. An example outcome of a Gibbs sampling process

By applying the rejection sampling principle, we take samples of the UE orientation $\theta^{(i)}$ and the likelihood target set as

$$
\theta^{(i)} \sim \mathcal{U}(-\pi / 2, \pi / 2) \text { and } \rho \sim \mathcal{U}(0,1) .
$$

The resulting UE orientation sample is accepted, if

$$
\rho<p\left(\left\{\hat{\boldsymbol{\eta}}_{k} \mid k \in \Theta_{\text {allpaths }}\right\} \mid \theta^{(i)}, \mathbf{p}_{\mathrm{SC}, k}^{(i)}, \mathbf{p}_{\mathrm{RX}}^{(i)}\right),
$$

where the value of the likelihood function is evaluated by substituting $\breve{\theta} \leftarrow \theta^{(i)}$ in the likelihood function given in (26). Again, if the condition in (31) is not valid, new samples are taken based on (30) until the condition is fulfilled.

\section{Numerical EVAluations AND ANALysis}

For the simulations, we assume a single BS scenario, where the BS is located at the origin $\mathbf{p}_{\mathrm{TX}}=[0,0]^{T}$ with a zero antenna orientation, and the UE position at $\mathbf{p}_{\mathrm{RX}}=[42,5]^{T}$ (in meters) with the antenna orientation of $\theta=21 \mathrm{deg}$. With the considered single BS scenario, the network synchronization requirements can be alleviated, and furthermore, it is not necessary to presume coverage from multiple BSs at the same time, which can be challenging at the mm-wave band with high path losses. The number of transmit antennas (in BS) and receive antennas (in UE) are determined as $N_{\mathrm{T}}=32$ and $N_{\mathrm{R}}=8$, and the number of digital paths is defined as $N_{b}=1$. The signal transmitted by the BS consists of $M=32$ OFDM symbols with Quadrature Phase Shift Keying (QPSK) modulated subcarriers. The beamforming matrix $\mathbf{F}[m, n]$ is defined so that the beam directions are uniformly distributed from $-\pi / 2$ to $\pi / 2$ radians over the transmitted OFDM symbols $m=0, \ldots, M-1$ with joint beamforming across all subcarriers $n$. Based on the 5G specifications in [15], the sampling rate is chosen as $245.76 \mathrm{MHz}$, which corresponds to $120 \mathrm{kHz}$ subcarrier spacing with FFT-size of 2048. Moreover, by taking into account the required minimum guardband, specified in [16], we assume $N=64$ uniformly distributed reference subcarriers in the active band to be available for the positioning purposes. In addition, we define the carrier frequency as $f_{c}=38 \mathrm{GHz}$ according to the allocated 5G mm-wave band given in [16]. 


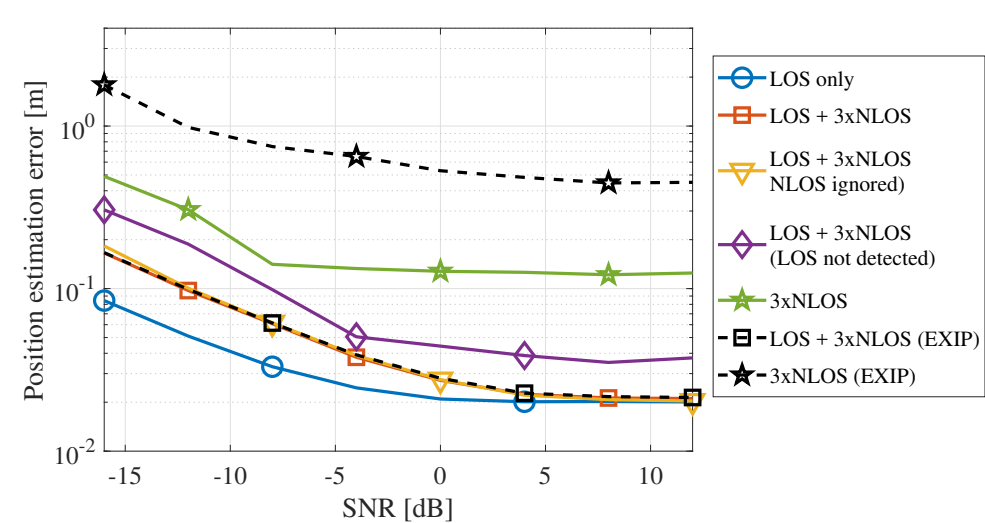

(a)

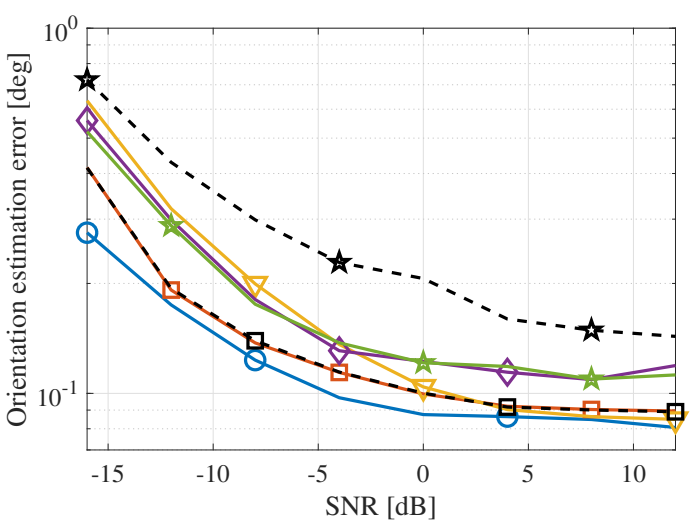

(b)

Fig. 3. The average (a) position estimation error and (b) orientation estimation error of the proposed Gibbs-sampling-based approach and corresponding reference results based on the EXIP approach (dashed lines) for the considered scenarios.

All radio paths are attenuated based on a free-space loss model with additional $6 \mathrm{~dB}$ reflection or scattering losses assumed for the NLOS paths.

The estimation of AOD and AOA is based on $N_{\text {fine }}=5$ fine-tuning iterations, as described in Algorithm 2. After this, the number of samples taken during the UE position and orientation estimation, summarized in Algorithm 3, is defined as $N_{\text {samp }}=2000$, where $N_{\text {burn-in }}=200$ first samples are discarded in order to reduce the effect of initialization. All provided results are averaged over 1000 independent trials.

We consider three separate radio propagation scenarios: a scenario with only the LOS path ("LOS only"), a scenario with the LOS path and 3 NLOS paths ("LOS+3xNLOS") with scatterer positions $\mathbf{p}_{\mathrm{SC}, 0}=[23,16]^{T}, \mathbf{p}_{\mathrm{SC}, 1}=[10,10]^{T}$, and $\mathbf{p}_{\mathrm{SC}, 2}=[14,-21]^{T}$ (in meters) as illustrated in Fig. 2, and a scenario with the 3 NLOS paths without the LOS path ("3xNLOS"). In addition, regarding the LOS $+3 x$ NLOS scenario, we study two additional use case conditions, which have substantial significance in practical implementations. In the first one, in order to reveal the impact of NLOS path estimation on the UE position and orientation estimation, we simply ignore the NLOS paths and use only the LOS path in the estimation process ("LOS $+3 \times$ NLOS(NLOS ignored)"). This is different from the LOS only scenario, as the presence of multiple propagation paths affects the channel parameter estimation. In the second use case condition for the LOS $+3 x$ NLOS scenario, we assume that the LOS path is not detected as a LOS path ("LOS+3xNLOS(LOS not detected)") and the proposed estimation method is forced to treat the LOS path as a NLOS path. Thus, during the estimation process, an additional scatterer for the LOS path is introduced and typically located close to the line between the BS and UE.

In Fig. 3, the average UE position estimation errors and UE orientation estimation errors are shown for the considered scenarios over different values of SNR, which is defined as a ratio between the total received signal power before adding the noise and the total noise power over all utilized subcarriers.
In addition, for comparison, results based on a least squares approach with extended invariance principle (EXIP), utilized in [9], are provided for the LOS $+3 \times$ NLOS and $3 \times$ NLOS scenarios. The EXIP approach uses an iterative LevenbergMarquardt algorithm (LMA), which is able to converge towards locally optimum estimates. When the LOS path is available, the parameter initialization in LMA is straightforward, but otherwise, as proposed in [9], the LMA must run over up to hundreds of different parameter initialization steps resulting in increased in computational complexity. The initialization of the EXIP algorithm in this paper is implemented based on the description provided in [9]. Furthermore, whereas the EXIP approach provides only the parameter estimates, the proposed Gibbs sampling approach in this paper provides the joint parameter distribution, as well as the marginal distributions of each unknown parameter. As expected, the best positioning performance, shown in Fig. 3, is obtained with the LOS only scenario, since all signal power is concentrated on a single path and the number of unknown parameters is minimized. From the position error point of view, it seems that considering the NLOS paths in the estimation process does not have a significant effect on the positioning accuracy. In case that the LOS path is not detected, the positioning error is slightly increased compared to the case with LOS detection. However, compared to the $3 \times$ NLOS scenario, the LOS $+3 \times$ NLOS(LOS not detected) scenario is able to introduce a distinct improvement in the positioning accuracy. Furthermore, whereas the leastsquares-based EXIP algorithm provides similar performance with the LOS $+3 x$ NLOS scenario, it clearly loses in accuracy without the LOS path availability in the $3 x$ NLOS scenario. Due to the finite resolution of channel estimates, the position accuracy of all scenarios has an error floor, which limits the performance with high SNR values.

As shown in Fig. 3, the LOS $+3 x$ NLOS scenario has a better orientation estimation accuracy compared to the LOS $+3 x$ NLOS(NLOS ignored) scenario, which indicates that including the NLOS paths in the estimation process im- 


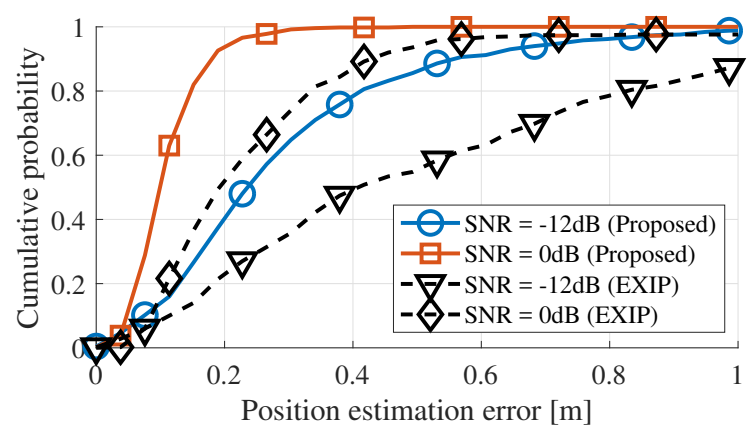

Fig. 4. Cumulative UE position estimation error of the proposed Gibbssampling-based approach and reference results based on the EXIP approach (dashed lines) for the $3 \times$ NLOS scenario with separate SNR values.

proves the UE orientation estimation accuracy, especially with low SNR values. Moreover, as the UE orientation estimation accuracy is almost identical between the $3 x$ NLOS and LOS $+3 \times$ NLOS(LOS not detected) scenarios, it is obvious that the UE orientation estimation does not benefit from the LOS path unless it is properly detected. When comparing the orientation estimation accuracy of the proposed Gibbs sampling approach with the EXIP approach, there is a clear improvement for the proposed approach in the 3 XNLOS scenario.

In Fig. 4, a cumulative distribution of the UE position error is shown for the $3 \mathrm{xNLOS}$ scenario with two separate SNR values. It can be seen that the proposed Gibbs sampling approach outperforms the EXIP approach. Moreover, with $\mathrm{SNR}=0 \mathrm{~dB}$ the positioning error of the proposed approach is less than $22 \mathrm{~cm}$ for $95 \%$ of the time. The corresponding cumulative error for the UE orientation is shown in Fig. 5. Again the proposed Gibbs sampling approach is able to improve the estimation accuracy compared to the EXIP approach. With $\mathrm{SNR}=0 \mathrm{~dB}$, the proposed approach achieves orientation estimation accuracy less than $0.3 \mathrm{deg}$ for $95 \%$ of the time.

\section{CONCLUSION}

In this paper, we studied UE position and UE orientation estimation in mm-wave $5 \mathrm{G}$ systems by considering a single BS scenario. Based on the mm-wave channel sparsity, we presented methods for estimating the AOD, AOA and TOA, for a multipath scenario with and without the presence of a LOS path. After this, we proposed using an iterative Gibbs sampling for obtaining joint statistical descriptions of the unknown UE position, UE orientation, and scatterer positions of each NLOS path. For this, we analytically derived the conditional distributions, from which each unknown parameter can be iteratively sampled according to the Gibbs sampling principle.

The presented results show that the proposed approach is able to reach cm-level UE positioning accuracy with less than $1 \mathrm{deg}$ orientation estimation error. Based on the results, the availability of the LOS path increased the UE position and UE orientation estimation accuracy. By considering also the NLOS paths, the accuracy of UE orientation estimation was further improved. Moreover, it was shown that the proposed approach

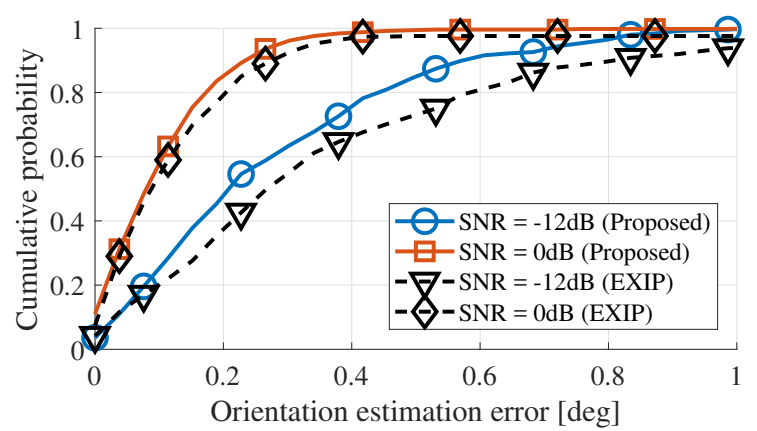

Fig. 5. Cumulative UE orientation estimation error of the proposed Gibbssampling-based approach and reference results based on the EXIP approach (dashed lines) for the $3 \times$ NLOS scenario with separate SNR values.

was able to perform without a separate LOS detection, and yet to improve the position estimation accuracy compared to the scenario with NLOS paths only.

\section{REFERENCES}

[1] M. Agiwal, A. Roy, and N. Saxena, "Next Generation 5G Wireless Networks: A Comprehensive Survey," IEEE Commun. Surveys Tuts., vol. 18 , no. 3, pp. 1617-1655, thirdquarter 2016.

[2] Z. Marzi, D. Ramasamy, and U. Madhow, "Compressive Channel Estimation and Tracking for Large Arrays in mm-Wave Picocells," IEEE J. Sel. Topics Signal Process., vol. 10, no. 3, pp. 514-527, April 2016.

[3] N. Garcia et al., "Location-aided mm-wave channel estimation for vehicular communication," in 2016 IEEE 17th Int. Workshop on Signal Process. Advances in Wireless Commun., July 2016, pp. 1-5.

[4] G. Destino, M. Juntti, and S. Nagaraj, "Leveraging sparsity into massive MIMO channel estimation with the adaptive-LASSO," in 2015 IEEE Global Conf. on Signal and Inf. Process., Dec 2015, pp. 166-170.

[5] Z. Guo, X. Wang, and W. Heng, "Millimeter-Wave Channel Estimation Based on Two-Dimensional Beamspace MUSIC Method," IEEE Trans. Wireless Commun., vol. PP, no. 99, pp. 1-1, 2017.

[6] H. Deng and A. Sayeed, "Mm-wave MIMO channel modeling and user localization using sparse beamspace signatures," in IEEE 15th Int. Workshop on Signal Process. Advances in Wireless Commun., June 2014, pp. 130-134.

[7] K. Witrisal et al., "High-Accuracy Localization for Assisted Living: 5G systems will turn multipath channels from foe to friend," IEEE Signal Process. Mag., vol. 33, no. 2, pp. 59-70, March 2016.

[8] J. Saloranta and G. Destino, "On the utilization of MIMO-OFDM channel sparsity for accurate positioning," in 2016 24th European Signal Process. Conf., Aug 2016, pp. 748-752.

[9] A. Shahmansoori et al., "Position and Orientation Estimation Through Millimeter-Wave MIMO in 5G Systems," IEEE Trans. Wireless Commun., vol. 17, no. 3, pp. 1822-1835, March 2018.

[10] J. Talvitie et al., "Novel Algorithms for High-Accuracy Joint Position and Orientation Estimation in 5G mmWave Systems," in 2017 IEEE Globecom Workshops, Dec 2017, pp. 1-7.

[11] R. Mendrzik et al., "Harnessing nlos components for position and orientation estimation in $5 \mathrm{~g}$ millimeter wave mimo," IEEE Trans. on Wireless Commun., vol. 18, no. 1, pp. 93-107, Jan 2019.

[12] J. H. Brady and A. M. Sayeed, "Wideband communication with highdimensional arrays: New results and transceiver architectures," in 2015 IEEE Int. Conf. on Commun. Workshop, June 2015, pp. 1042-1047.

[13] M. F. Duarte et al., "Distributed Compressed Sensing of Jointly Sparse Signals," in Conf. Rec. of the Thirty-Ninth Asilomar Conf. on Signals, Syst. and Comput., 2005., October 2005, pp. 1537-1541.

[14] K. Murphy, Machine Learning: A Probabilistic Perspective, ser. Adaptive computation and machine learning. MIT Press, 2012.

[15] 3GPP TS 38.211 V15.1.0, "NR Physical channels and modulation (Rel. 15)," Mar. 2018.

[16] 3GPP TS 38.101-2 V15.1.0, "User Equipment (UE) radio transmission and reception; Part 2: Range 2 Standalone (Rel. 15)," Mar. 2018. 